\title{
Review Article \\ Functions of the Tumor Suppressors p53 and Rb in Actin Cytoskeleton Remodeling
}

\author{
Takahiro Ebata, ${ }^{1}$ Hiroaki Hirata, ${ }^{2}$ and Keiko Kawauchi ${ }^{1,3}$ \\ ${ }^{1}$ Frontiers of Innovative Research in Science and Technology, Konan University, 7-1-20 Minatojima-minamimachi, \\ Chuo-ku, Kobe, Hyogo 650-0047, Japan \\ ${ }^{2}$ Nagoya University Graduate School of Medicine, 65 Tsurumai, Showa-ku, Nagoya, Aichi 466-8550, Japan \\ ${ }^{3}$ Department of Molecular Oncology, Institute for Advanced Medical Sciences, Nippon Medical School, 1-396 Kosugi-cho, \\ Nakahara-ku, Kawasaki, Kanagawa 211-8533, Japan \\ Correspondence should be addressed to Keiko Kawauchi; kawauchi@center.konan-u.ac.jp
}

Received 8 September 2016; Accepted 21 November 2016

Academic Editor: Wuyuan Lu

Copyright ( 2016 Takahiro Ebata et al. This is an open access article distributed under the Creative Commons Attribution License, which permits unrestricted use, distribution, and reproduction in any medium, provided the original work is properly cited.

\begin{abstract}
Mechanical microenvironments, such as extracellular matrix stiffness and strain, have crucial roles in cancer progression. Cells sense their microenvironments with mechanosensing biomolecules, which is accompanied by the modulation of actin cytoskeleton structures, and the signals are subsequently transduced downstream as biochemical signals. The tumor suppressors p53 and retinoblastoma protein $(\mathrm{Rb})$ are known to prevent cancer progression. The $\mathrm{p} 53$ and $\mathrm{Rb}$ signaling pathways are disrupted in many types of cancers. Here, we review recent findings about the roles of these tumor suppressors in the regulation of mechanosensing biomolecules and the actin cytoskeleton. We further discuss how dysfunction in the p53- and/or Rb-mediated mechanosignaling pathways is potentially involved in cancer progression. These pathways might provide good targets for developing anticancer therapies.
\end{abstract}

\section{Introduction}

During cancer progression, cells acquire several abilities, including continual unregulated proliferation, resistance to cell death, invasiveness, and epithelial-mesenchymal transition (EMT) [1-3]. Remodeling of the actin cytoskeleton is also associated with cancer progression $[4,5]$. Actin is one of the most abundant proteins in eukaryotic cells. Globular actin (G-actin) monomers polymerize into actin filaments (F-actin), which is then depolymerized, in a steadystate equilibrium. Actin polymerization is regulated by actin nucleators, including the formins, actin-related protein $2 / 3$ (Arp2/3) complex, and spire [6-8]. The activation of these actin nucleators is regulated by Rho GTPases, including Rho, $\mathrm{Rac}$, and Cdc42, which typically induce the formation of stress fibers, lamellipodia, and filopodia, respectively [9]. Actin depolymerization is enhanced by gelsolin and actindepolymerizing factor (ADF)/cofilin, while spontaneous depolymerization is slow [10]. Gelsolin is activated by calcium ions but inhibited by phosphatidylinositol 4,5-bisphosphate. Activation of $\mathrm{ADF} /$ cofilin is regulated by its phosphorylation. Kinases, including LIM kinase (LIMK), testicular protein kinase (TESK), and integrin-linked kinase (ILK), induce the activation of $\mathrm{ADF} /$ cofilin, while phosphatases, such as slingshot and chronophin, induce its inactivation [11-13].

The invasion of cancer cells is associated with the formation of several actin-mediated structures, including lamellipodia, filopodia, podosomes, and invadopodia [14, 15]. Podosomes and invadopodia degrade the extracellular matrix (ECM), which facilitates invasion into other tissues. Protrusions of lamellipodia and filopodia are likely to promote cancer cell invasion through the generation of traction forces that are required for mesenchymal-mode migration. Blebs are also formed during the migration of cancer cells and promote their invasion $[16,17]$. The formation of blebs is initiated upon disruption of the actin cortex and driven by intracellular pressure generated in the cytoplasm. In association with reassembly of the actin cortex, the blebs are then retracted by 
actomyosin contraction, generating traction forces that move the cells forward. While membrane blebbing is typically associated with apoptotic cell death [18], aggressive cancer cells appear to form and use blebs for invasion independently of cell death $[16,17]$. In addition to invasion, the insensitivity of aggressive cancer cells to antitumor drug-induced apoptosis might also be affected by actin cytoskeletal structures. Stress fibers, lamellipodia, and filopodia are considered to promote the survival of cancer cells.

Actomyosin contraction is essential for sensing the mechanical environments surrounding cells [19]. At the sites of cell-ECM adhesion, the formation of focal adhesion complexes, including integrins, focal adhesion kinase (FAK), p130 Crk-associated substrate (p130Cas; also known as Bcar1), and paxillin, promotes actin polymerization and activates myosin [20-24]. Activated actomyosin generates a contractile force that induces conformational changes in several focal adhesion proteins to enhance downstream signaling [25]. Furthermore, $\alpha$-catenin has been identified as a mechanosensing protein in adherens junction (AJ) complexes at cellcell junctions [26]. The homophilic interaction of cadherin ectodomains induces the assembly of AJ complexes and local actin polymerization. While actin filaments link to cadherins via $\beta$-catenin and $\alpha$-catenin, an actomyosin-generated force transmitted to this linkage causes conformational changes in $\alpha$-catenin, which promotes its binding to vinculin [27]. This results in the recruitment of various proteins, including zyxin and Arp2/3, to AJs and a further increase in actin polymerization $[26,28]$. The expression and activity of proteins in focal adhesions and $\mathrm{AJ}$ complexes are often altered during cancer progression.

Actin dynamics influence cellular behavior not only by regulating cytoskeletal organization but also by controlling gene expression. For example, in skeletal muscle differentiation, disassembly of actin filaments is required for the muscle-specific gene expression induced by serum response factor (SRF) [29-32]. G-actin binds to megakaryocytic acute leukemia (MAL; also known as MKL1/MRTF-A), a cofactor of SRF, and sequesters it from the nucleus, therefore, causing alterations in the equilibrium between actin polymerization and depolymerization perturb differentiation [33].

Cancer progression is associated with the accumulation of gene mutations and cancer is generally considered to be a genomic disease. TP53, which encodes the p53 transcription factor, is mutated in more than $50 \%$ of human cancers [34]. This protein exerts its biological activities, such as cell cycle arrest and induction of apoptosis or senescence, by upregulating the expression of various target genes [35-37]. Stresses, such as DNA damage, induce the stabilization and activation of p53 by affecting its posttranscriptional modifications such as phosphorylation and acetylation $[38,39]$. The expression level of p53 is low under low stress conditions. However, it still contributes to cellular homeostasis involving differentiation and cell cycle progression.

Germline mutations in the retinoblastoma $(R b)$ gene occur frequently in retinoblastoma, which is the most common cancer of the developing retina in early childhood [40]. Somatic mutations in $\mathrm{Rb}$ are also observed in several cancers, including small-cell lung cancer and bladder cancer [41].

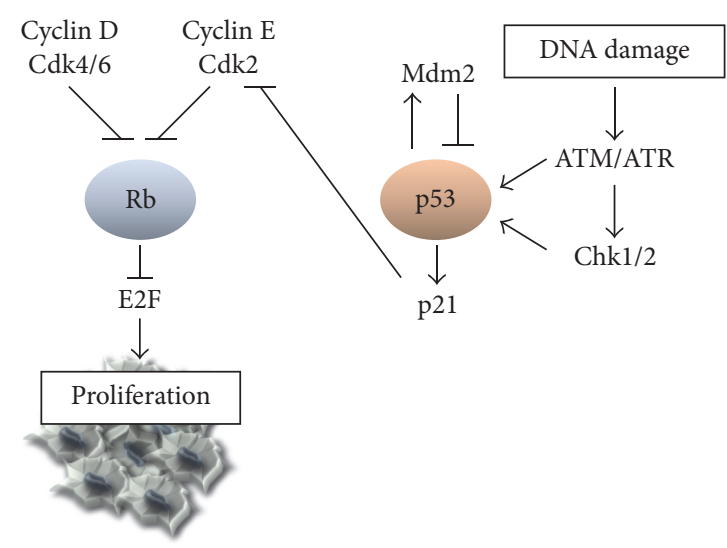

FIGURE 1: p53 suppresses the cell proliferation mediated by the Rb-E2F pathway. Phosphorylation of Rb by CDK4/6-cyclin D and $\mathrm{CDK} 2$-cyclin $\mathrm{E}$ causes the dissociation of $\mathrm{Rb}$ from $\mathrm{E} 2 \mathrm{~F}$ to promote cell cycle progression. In response to DNA damage, ataxia telangiectasia mutated (ATM) or ataxia telangiectasia and Rad3-related protein (ATR) are activated and phosphorylate p53 either directly or through Chk1/2. Phosphorylated p53 dissociates from Mdm2 and is thereby stabilized. Active p53 then induces the transcription of its target genes involving $p 21^{W A F 1}$, resulting in the inhibition of CDK2cyclin E activity.

Furthermore, inactivation of Rb by hyperphosphorylation via the constitutive activation of its kinases has been implicated in tumor initiation and progression [40, 42, 43]. Phosphorylation of $\mathrm{Rb}$ by cell cycle kinase complexes, that is, cyclindependent kinase (CDK) 4/6-cyclin D and Cdk-2-cyclin E, releases the transcriptional repression of $\mathrm{E} 2 \mathrm{~F}$, resulting in cell cycle progression. The activity of Cdk-2-cyclin E is inhibited by the CDK-inhibitor p $21^{\text {WAF1 }}$, a transcriptional target of p53 (Figure 1) [44]. Rb also plays a critical role in the development of several tissues, including muscle and bone, by regulating other transcriptional factors such as MyoD and RUNX2 [43].

While various molecules that constitute the actin cytoskeleton, focal adhesions, and AJs are involved in sensing the mechanical microenvironments surrounding cells, little is known about the contribution of $\mathrm{p} 53$ and $\mathrm{Rb}$ to mechanosensing. In this review, we summarize the roles of p53 and Rb in the regulation of the actin cytoskeleton and mechanosensing proteins, which provides insights into the mechanisms of cancer progression.

\section{2. p53 Regulates Integrin Expression and Activation}

Integrins, which are heterodimers composed of $\alpha$ and $\beta$ subunits, form a connection between the ECM and actin cytoskeleton, and their downstream signaling molecules drive actin polymerization [45-47]. In humans, 18 types of $\alpha$ subunits and 8 types of $\beta$ subunits have been identified, and they assemble into 24 types of integrins that bind specifically to their ligands, including fibronectin, laminin, and collagen [48]. The binding of a ligand to the extracellular domain of integrin induces the recruitment of focal adhesion proteins, including FAK, p130Cas, and paxillin, at the cytoplasmic side, 


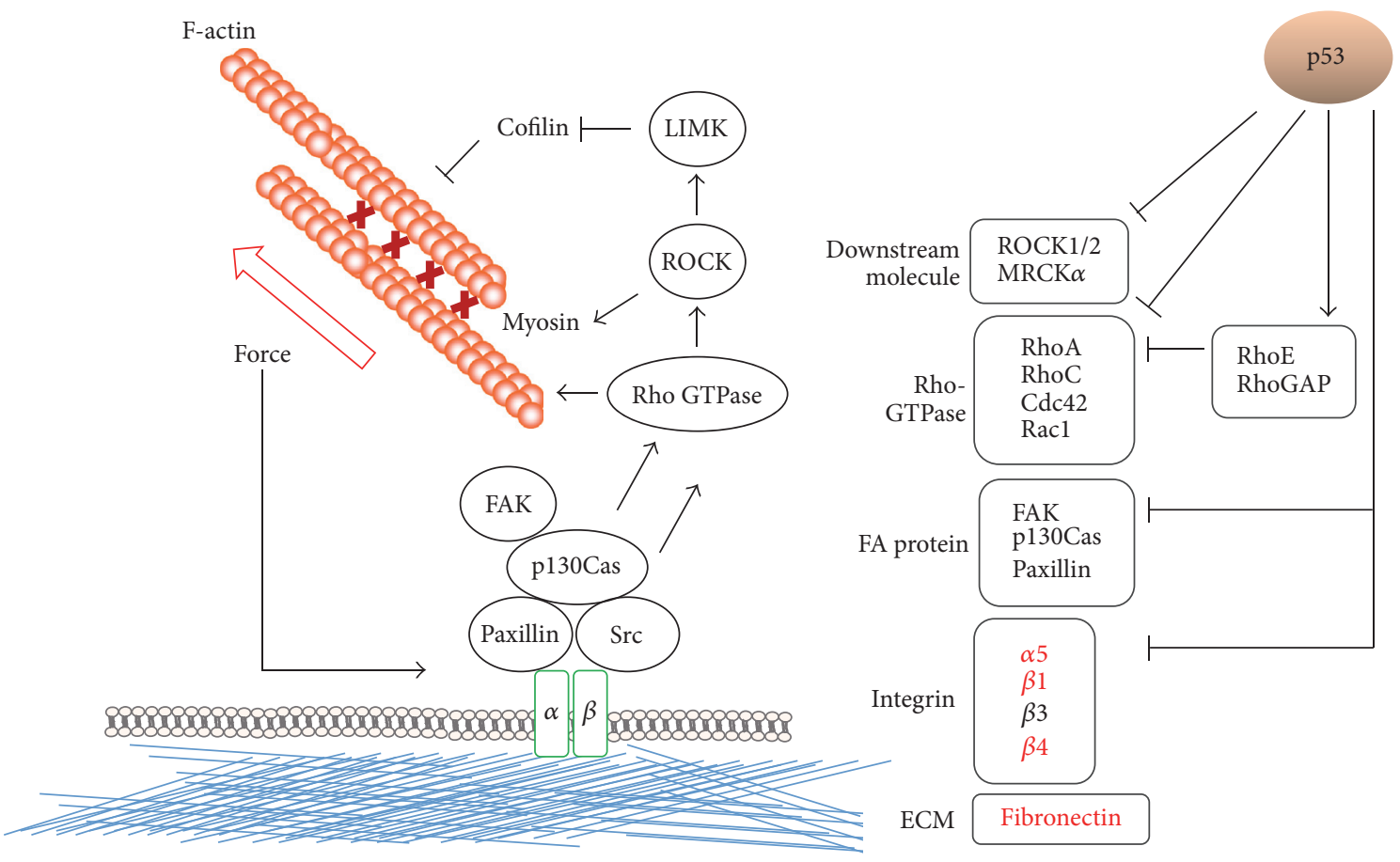

FIGURE 2: Effects of p53 on molecules in mechanosensing pathways. Cells sense ECM stiffness and modulate actin cytoskeleton structures through the integrin signaling pathways. p53 wild-type suppresses cancer progression through downregulation of the molecules in the integrin signaling pathways. Conversely, several p53 mutants exert gain-of-function effects on the upregulation of molecules in the integrin signaling pathways. The molecules indicated by red letters are upregulated by $533 \mathrm{GOF}$ mutants.

leading to the activation of Rho GTPases and stimulation of actin polymerization [20-24]. The cytoplasmic domain of integrin links to actin filaments through several adaptor proteins. Actomyosin contraction potentially modulates the affinity of integrin to its ligands by changing the conformation of integrin.

Integrin signaling plays a fundamental role in tumor cell invasion and metastasis. The expression levels of several integrins, including $\alpha 5 \beta 1, \alpha 6 \beta 4, \alpha 4 \beta 1, \alpha \mathrm{v} \beta 3, \alpha \mathrm{v} \beta 5, \alpha \mathrm{v} \beta 6$, and $\alpha 2 \beta 1$, in cancer cells correlate with their aggressiveness [46, $49,50]$. It has been revealed that $\mathrm{p} 53$ regulates the expression of integrins $\alpha 5, \beta 1, \beta 3$, and $\beta 4$ (Figure 2) [51-56]. Janouskova et al. showed that Nutlin-3a, an MDM2 antagonist that acts as a p53 activator, decreases the expression of integrin $\alpha 5$ in glioma and colorectal cancer cells $[51,52]$. Bon et al. reported that the expression of integrin $\beta 4$ was decreased by either the ectopic expression of p53 or DNA damage in wild-type p53expressing cells [56]. Conversely, the ectopic expression of transactivating p63 (TAp63) or transactivating p73 (TAp73), two 553 family members, increased the promoter activity of ITGB4, which encodes integrin $\beta 4$. Importantly, depletion of p53 enhanced the TAp63- or TAp73-dependent activation of the ITGB4 promoter. Not only DNA damage triggered by genotoxic drugs or activation of oncogenes but also other forms of stress, such as chromosomal aberrations, hypoxia, and telomere shortening, are associated with cancer progression [36]. Repression of integrin $\alpha 5$ and $\beta 4$ expression by $\mathrm{p} 53$ activated in response to these stresses is likely to prevent the progression of cancer stimulated by these stresses.

Vaillant et al. reported, using mammary tumors derived from p53-deficient mice lacking one allele $\left(p 53^{+/-}\right)$, that loss of $\mathrm{p} 53$ function promotes cancer cell invasion by upregulating integrin $\beta 3$ expression at the cell surface [54]. We also reported that the depletion of $\mathrm{p} 53$ increased the expression of integrin $\beta 3$, encoded by ITGB3, in a transcription factor NF$\kappa \mathrm{B}$-dependent manner. This leads to an increase in integrin $\alpha \mathrm{v} \beta 3$ expression at the cell surface, which promotes the formation of lamellipodia. Lamellipodia formation mediated by integrin $\alpha \mathrm{v} \beta 3$ contributes to the constitutive activation of another transcription factor, STAT3, which plays an integral role in tumor cell invasion [55]. Conversely, Qui et al. showed that pifithrin- $\alpha$, a p53 inhibitor, increases the expression of integrin $\beta 1$ in endothelial cells when the expression of ID1 (encoding inhibitor of DNA binding [ID] 1, which belongs to a family of basic helix-loop-helix transcription factors lacking DNA-binding domains and plays a critical role in angiogenesis) is depleted [53]. Pifithrin- $\alpha$ also enhances the formation of F-actin at the peripheral rim and promotes tubular formation. ID1 expression is upregulated in angiogenic tumor vessels. These results suggest that the p53-dependent maintenance of the low expression levels of integrins $\beta 1$ and $\beta 3$ helps to attenuate both cancer cell invasion and angiogenesis, which would prevent cancer progression. 


\section{Regulation of Focal Adhesion-Rho Signaling Pathways by $\mathbf{p 5 3}$}

FAK contains three distinct domains: a four-point-one, ezrin, radixin, moesin (FERM) domain; a kinase domain; and a focal adhesion targeting (FAT) domain [21]. The residues in the FERM domain are responsible for the autoinhibition of FAK by intramolecular interactions. External forces are believed to induce a conformational change of the FAT domain to disrupt these intramolecular interactions. However, since FAK does not bind directly to actin filaments, it is unclear whether FAK activity in cells is truly regulated by force such as actomyosin contraction.

Phosphorylation of p130Cas is also facilitated by external forces [57]. Src phosphorylates the substrate domain of p130Cas (CasSD), which is characterized by 15 YXXP motifs. Phosphorylated CasSD provides a binding site for the CrkDOCK180 complex, a guanine nucleotide exchange factor (GEF) for Rac. External forces induce a conformational change of CasSD, which facilitates the phosphorylation of p130Cas. Like FAK, p130Cas does not bind directly to actin filaments [25]. Recently, we found that tensin 1 mediates the interaction of p130Cas with actin filaments [58]. However, given that the expression level of tensin 1 is generally low in metastatic cancers $[59,60]$, it may not be responsible for the enhanced phosphorylation of p130Cas and FAK in aggressive cancer cells.

Paxillin, an adaptor protein at focal adhesions, is known to be involved in the mechanical-cue-dependent regulation of Rho GTPases [22]. While the underlying mechanism remains unclear, the C-terminal LIM domains of paxillin are likely to be involved in the responses of paxillin against mechanical inputs [61]. Paxillin potentially promotes the invasion of cancer cells; however, its levels of expression and phosphorylation differ largely among cancer cell types [62].

It has been suggested that p53 regulates these mechanoresponsive proteins at focal adhesions (Figure 2). The ectopic expression of p53 suppresses the promoter activity of FAK either directly [63] or by inducing the expression of X-linked ectodermal dysplasia receptor (XEDAR), a member of the tumor necrosis factor receptor (TNFR) superfamily [64]. Recently, we reported that oncogenic Ras-induced transformation leads to the cleavage of $\beta$-actin and concomitant suppression of p130Cas phosphorylation in a p53-dependent manner [65]. We have further shown that both the oncogenic Ras-induced disruption of mitochondrial integrity and p53mediated activation of the mitochondrial protease high temperature requirement $\mathrm{A} 2$ ( $\mathrm{Htr} \mathrm{A} 2$; also known as $\mathrm{Omi}$ ) are involved in the cleavage of $\beta$-actin in Ras-transformed cells. The p130Cas-Racl axis is known to promote cell invasion by inducing lamellipodia formation [66]. Interestingly, the cytoplasmic localization of p53 was increased by Ras transformation, causing mitochondrial translocation of the mitogen-activated protein kinase p38. p38 translocated into mitochondria and then enhances activation of HtrA2/Omi [67]. While cytoplasmic p53 is known to have tumor suppressive functions via the suppression of centrosome duplication, induction of cell death, and inhibition of autophagy [68], our results revealed a novel mechanism underlying the tumor suppressive function of cytoplasmic p53.

The constitutively active form of Src and oncogenic Ras induce cellular transformation and invasion. Mukhopadhyay et al. reported that loss of p53 function enhances Src-driven cell invasion by promoting the formation of actin-rich structures, such as podosomes and invadopodia [14, 69-71]. They showed that the level of caldesmon, an actin binding protein that can suppress both podosome and invadopodia formation [72], was decreased by inhibition of p53 [70]. Further, there is a possibility that p53 diminishes Src-driven cell invasion via inhibition of p130Cas-mediated podosome and invadopodia activity. As described above, a decrease in p130Cas phosphorylation by impairment of actin filaments following Rasinduced transformation was suppressed by p53 knockdown, while the activity of Src, a kinase of p130Cas, was not affected. Indeed, knockdown of p53 decreases p130Cas phosphorylation in Src-transformed cells (personal communication).

p53 regulates the activity of Rho GTPases (Figure 2) [73-75]. p53 mediates the oncogenic-Ras-induced activation of p190 Rho GTPase-activating protein (RhoGAP) [76]. A deficiency in p53 expression increases RhoA activity and stimulates the formation of blebs via the activation of Rhoassociated protein kinase (ROCK) [77]. While RhoA typically promotes stress fiber formation [9], RhoA can increase cellexerted contractile forces even in Ras-transformed cells in which stress fiber formation is largely diminished [76]. The generation of cell-exerted contractile forces plays a crucial role in the invasion and intravasation/extravasation of cancer cell tissue and blood vessels.

p53 regulates the expression of several genes that encode effector proteins of RhoA/RhoC and Cdc42. For example, depletion of p53 increases the expression of ROCK1/2 and $M R C K \alpha$, which encodes myotonic dystrophy kinase-related Cdc42-binding kinase $\alpha(\mathrm{MRCK} \alpha)$. The ectopic expression of p53 in turn increases the expression of these genes [78]. Not only RhoA signaling but also Rac and Cdc42 signaling is affected by p53. Depletion of p53 increases phosphoinositide 3-kinase- (PI3-kinase-) mediated Rac activity [79]. Conversely, the ectopic expression of p53 decreases Cdc42 activity and concomitant filopodia formation [80].

F-actin formation is both negatively and positively regulated by $\mathrm{p} 53$ in response to DNA damage. Croft et al. reported that treatment with the antitumor drug doxorubicin reduces the activity of cofilin by increasing the expression of RhoC and LIM kinase 2 (LIMK2) in a p53-dependent manner [81]. While doxorubicin treatment promotes the formation of stress fibers, depletion of either RhoC or LIMK2 abrogates doxorubicin-induced stress fiber formation. Conversely, other antitumor drugs, including camptothecin and etoposide, attenuate the formation of stress fibers through the p53dependent expression of RhoE [82]. Depletion of RhoE prevents the camptothecin-induced disassembly of stress fibers.

It has been suggested that, in response to DNA damage, p53 influences actin cytoskeleton remodeling by regulating the cytoskeleton adaptor protein ankyrin-1, which is encoded by $A N K 1$. Hall et al. reported that the etoposide-induced activation of p53 increases the expression of ANK1 [83]. Etoposide treatment promotes the formation of actin-rich long 
protrusions, even though knockdown of ankyrin-1 attenuates this response. By contrast, stress fiber formation in etoposidetreated cells is enhanced by depleting ankyrin-1 expression. The ankyrin-1-mediated activation of cofilin may be involved in these actin remodeling processes. Furthermore, ankyrin1 contributes to the association of the cortical spectrin-actin network with the plasma membrane by linking spectrin with membrane proteins including the anion exchanger and CD44 $[84,85]$. Spectrin plays a crucial role in maintaining the structural integrity of the plasma membrane and has been suggested to be a potential mechanosensing protein [86]. Therefore, the p53-dependent regulation of ankyrin1 may contribute not only to actin remodeling but also to mechanosensing/mechanoprotection of cells.

\section{Regulation of Cadherin Expression by p53}

It is well established that EMT promotes cell invasion and metastasis. It is important to note that EMT is associated with a decrease in E-cadherin expression and an increase in $\mathrm{N}$ cadherin expression, which are major components of cellcell adhesion complexes. They form homophilic adhesion bonds. Since the interactions of $\mathrm{N}$-cadherin are much weaker than those of E-cadherin, the shift from E-cadherin to $\mathrm{N}$ cadherin during EMT weakens cell-cell adhesions, which promotes the scattering and migration of cancer cells. Like focal adhesions, AJs are reportedly involved in sensing the mechanical microenvironments of cells [26, 87-90].

In cells undergoing EMT, the expression of E-cadherin, encoded by $C D H 1$, is suppressed by Snail, zinc finger E-box binding homeobox 1/2 (ZEB1/2), and Slug. These proteins in turn increase the expression of $\mathrm{N}$-cadherin, which is encoded by $\mathrm{CDH} 2$ [91]. It has been revealed that p53 prevents EMT by regulating the expression of $\mathrm{E}$ - and $\mathrm{N}$-cadherins. Siemens et al. showed that activated p53 suppresses Snail expression by inducing the expression of microRNA- (miR-) $34 \mathrm{a} / \mathrm{b} / \mathrm{c}$ [92]. The expression of miR-200 and miR-192 is also p53-dependent, and their expression is negatively correlated with ZEB1/2 expression [93, 94]. Both p53 itself and its transcriptional targets (MDM2 and $\left.\mathrm{p} 21^{\mathrm{WAF} 1}\right)$ regulate Slug expression [95]. The ectopic expression of p53 induces the proteasomal degradation of Slug, which is mediated by the E3 ligase MDM2. A further study by Kim et al. showed that complex formation of Slug with p2 $1^{\mathrm{WAF} 1}$ and $\mathrm{p} 53$ is involved in MDM2-mediated Slug degradation [96].

\section{5. p53 Gain-of-Function Mutants Regulate Cell Adhesion Molecules and Downstream Pathways}

Mutations in TP53 often result in a gain-of-function (GOF) of the protein [97]. Muller et al. showed that p53 GOF mutants increase the Rab-coupling protein- (RCP-) driven recycling of integrin $\alpha 5 \beta 1$ by inhibiting TAp63-mediated transcription [98]. This would induce the formation of filopodia-like protrusions and thereby promotes the invasion of cancer cells. Furthermore, p53 GOF mutants promote the translocation of integrin $\beta 1$ to the tips of filopodia by increasing the early

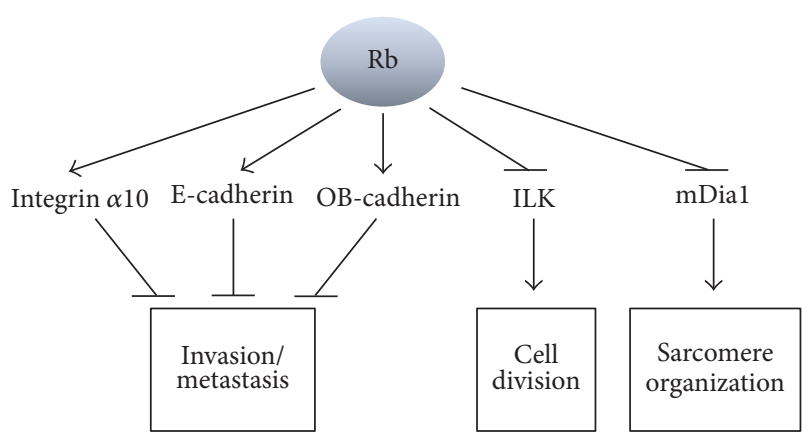

FIGURE 3: Rb-dependent regulation of the molecules involved in actin cytoskeleton remodeling. In cancer cells, $\mathrm{Rb}$ is inactivated either by Cdk4/6- and Cdk2-dependent hyperphosphorylation or by its mutation. $\mathrm{Rb}$ prevents cancer progression by suppressing both invasion and cell division via the alteration of actin cytoskeleton remodeling. Conversely, under inflammatory conditions, $\mathrm{Rb}$ is likely to have a promoting effect on cancer cachexia, which is associated with muscle atrophy characterized by disorganization of sarcomeres. $\mathrm{TNF} \alpha$ upregulates the Cdk4/6-dependent phosphorylation of $\mathrm{Rb}$, which causes disorganization of sarcomeres by inhibiting mDialmediated actin polymerization.

growth response protein 1- (EGR1-) mediated expression of myosin-X (Myo10), an unconventional myosin [99]. The expression of integrin $\alpha 5 \beta 1$ and its ligand fibronectin is also increased by p 53 GOF mutants [100], which contributes to the survival of suspended cells.

Recently, Lee et al. showed that p53 GOF mutants increase the transcription of ITGB4, which encodes integrin $\beta 4$ [101]. TAp63 and TAp73 also upregulate the expression of ITGB4, as described above [56]. Conversely, the transcriptional activity of TAp63 and TAp73 is reduced by their binding with a subset of p53 mutants [102]. Thus, additional studies are needed to reveal the actual relationship between p53 GOF mutants, p63, and p73 in terms of the regulation of ITGB4 expression, which would provide a better understanding of the mechanisms underlying cancer progression.

p53 GOF mutants appear to contribute to cancer progression via the expression of the EMT-promoting factors Snail, Slug, and Twist [103, 104]. In addition, p53 GOF mutants upregulate the expression of paxillin and ARHGDI, which encodes Rho GDP-dissociation inhibitor alpha (RhoGDI $\alpha$ ) that typically downregulates Rho GTPases [105]. However, the role of paxillin and Rho-GDIs in metastasis is controversial, which may reflect the diversity of cancers.

\section{Rb Regulates Cell Adhesion Molecules and Downstream Pathways}

Several studies have suggested a role for $\mathrm{Rb}$ in the regulation of the actin cytoskeleton and related pathways (Figure 3). Engel et al. showed, using Rb knockout cells and the ectopic expression of $\mathrm{Rb}$ mutants, that $\mathrm{Rb}$ promotes the expression of ITGA10 (encoding integrin $\alpha 10$ ) in osteoblasts independently of the canonical Rb-E2F pathway [106]. They used bioinformatic analysis to show that the expression of integrin $\alpha 10$ is downregulated in several tumors compared with normal 
tissues. Integrin $\alpha 10$ heterodimerizes with the integrin $\beta 1$ subunit [107]. While both loss-of-function and gain-of-function of p53 drive integrin $\beta 1$-mediated cancer cell progression as discussed above [53, 98-100], Rb might prevent cancer progression by increasing the heterodimerization of integrins $\alpha 10$ and $\beta 1$ and by suppressing the heterodimerization of integrins $\alpha 4$ or $\alpha 5$ with $\beta 1$.

It has been suggested that loss of $\mathrm{Rb}$ function causes the upregulation of ILK expression, which is required for cell division [108]. In retinoblastoma cells carrying mutations in the $R b$ gene, inhibition of ILK by the small molecule QLT-0267 induces the accumulation of multinucleated cells, which is associated with a decrease in cortical F-actin, alteration of mitotic spindle organization, and declustering of centrosomes. ILK upregulates actin polymerization by inactivating cofilin and by activating both Rac and Cdc42 [109]. Furthermore, ILK controls the complex formation of Aurora A kinase/chTOG/TACC3, which is essential for the assembly of mitotic spindles [110, 111]. Both microtubule-dependent forces and actin-dependent forces at the cell cortex contribute to centrosome clustering [112]. These findings suggest that loss of $\mathrm{Rb}$ function prevents mitotic defects, such as the arrest of and exit from mitosis, through upregulation of the ILK-mediated assembly of actin filaments and microtubules, which would ensure cell division.

We reported recently that $\mathrm{Rb}$ causes disruption of the sarcomeric structure of skeletal muscle myotubes via its interaction with the formin protein mDial [113], an effector of RhoA [114]. This pathway is stimulated by the inflammatory cytokine TNF $\alpha$. TNF $\alpha$ increases the CDK4-dependent but CDK2-independent phosphorylation of Rb. Phosphorylated $\mathrm{Rb}$ subsequently translocates from the nucleus to the cytosol, where it binds to mDial. The sarcomere, which consists of a highly ordered array of actin thin filaments and myosin thick filaments, provides a contractile unit in muscle cells and confers muscle strength. Our findings suggest that inflammation hampers the homeostasis of skeletal muscle via inhibition of mDial-mediated actin polymerization by $\mathrm{Rb}$. In advanced cancer patients, cachexia-the loss of body mass that is associated with muscle atrophy characterized by disorganization of sarcomeres-is often observed. Cytoplasmic Rb might contribute to cancer progression by promoting muscle atrophy. Indeed, cytoplasmic Rb was detected in atrophied tibialis anterior muscles, but not the normal muscles, of cancer patients [113].

$\mathrm{Rb}$ has also been implicated in the regulation of cadherin expression. Sosa-García et al. reported that, in Rb-deficient osteoblasts, the expression of E-cadherin and osteoblastcadherin (OB-cadherin) is downregulated [115]. Associated with this, AJs are disrupted in these cells, which is mediated by the inactivation of merlin, a member of the ezrin, radixin, and moesin (ERM) family of proteins that links actin filaments to AJ complexes [116]. OB-cadherin (also known as cadherin-11), a cadherin isoform that is expressed in mesoderm-derived tissues, is known to be involved in cancer progression both positively and negatively. In prostate and brain cancers, OB-cadherin enhances the engagement between cancer cells and bone tissues by its hemophilic interactions, which promotes cancer metastasis [117-122].
By contrast, osteosarcoma formation is promoted by the disruption of OB-cadherin-mediated cell-cell interactions. These results imply that loss of $\mathrm{Rb}$ function might induce cancer progression preferentially in growing tissues.

\section{Concluding Remarks}

In this paper, we focused on the findings regarding the roles of the central tumor suppressors $\mathrm{p} 53$ and $\mathrm{Rb}$ in the regulation of the actin cytoskeleton and mechanoresponsive molecules. Intense studies have revealed that both these tumor suppressors and mechanical environments surrounding cells have significant effects on cancer progression. However, little is known about how these tumor suppressors influence the mechanical environment-dependent regulation of cancer progression.

Cancer cells sense various different mechanical environments during metastasis, leading to an alteration of cell behavior $[123,124]$. The tumor stroma is composed of noncancerous cells, including cancer-associated fibroblasts (CAFs), as well as noncellular components, such as the ECM. The excess production of the ECM mainly by CAFs stiffens solid tumors $[125,126]$, which promotes the growth of cancer cells $[127,128]$. In addition, along with the growth of tumor mass, cancer cells suffer high pressure caused by tissue compression and/or an increase in interstitial pressure [129]. Similar to ECM stiffening, this high pressure also promotes the metastasis of cancer cells. Following intravasation, cancer cells in blood vessels are exposed to shear forces exerted by blood flow, which facilitates the interaction of cancer cells with endothelial cells to permit extravasation [130]. Cell growth and sensitivity against chemotherapy and radiotherapy treatments are also affected by mechanical environments [131]. Furthermore, mechanical environments appear to be crucial for maintaining the properties of cancer stem cells, such as self-renewal and tumor formation abilities. Thus, mechanical environments affect cancer progression at multiple stages.

We have proposed the possibility that cancer cachexia with muscle atrophy is caused by $\mathrm{Rb}$-mediated disruption of sarcomeric organization [113]. Currently there is no effective treatment for muscle atrophy, which makes it difficult to improve the quality of life (QOL) of patients with advanced cancers. Since CDK4 is responsible for the TNF $\alpha$-induced phosphorylation of $\mathrm{Rb}$ and resultant disorganization of sarcomeres, disrupting the function of CDK4 may prevent cachexia in advanced cancers, which would improve QOL. p53 is known to be required for the promotion of muscle atrophy induced by inflammatory cytokines, including TNF $\alpha$ [132]. p53 may be involved in Rb-promoted muscle atrophy. It is well known that the $\mathrm{p} 53$ and $\mathrm{Rb}$ pathways cooperatively regulate cell proliferation and senescence; however, the relationship between p53 and Rb in actin cytoskeleton remodeling is totally unknown. As described above, we have shown that $\mathrm{Rb}$ appears to inhibit mDial-mediated actin polymerization to disrupt sarcomeres. However, activation of RhoA, which promotes mDial-induced actin polymerization [114], is prevented by p53 [77]. These observations support the notion that p53 synergistically enhances $\mathrm{Rb}$-promoted 
muscle atrophy. In addition to CDK4 inhibition, development of a drug that targets the pathway for p53-mediated inactivation of RhoA may enable the suppression of cancer-associated cachexia.

While accumulating evidence reveals that mechanical environments significantly affect the aggressiveness of cancer cells, it remains unclear how mechanical environments regulate the activities of $\mathrm{p} 53$ and $\mathrm{Rb}$. Further studies into the mechanotransduction mechanisms responsible for mechanical cue-dependent regulation of these tumor suppressors would aid the development a definitive treatment for cancers and a treatment that improves the QOL of advanced cancer patients.

\section{Competing Interests}

The authors declare that there is no conflict of interests regarding the publication of this paper.

\section{Acknowledgments}

The authors thank Drs. Steven J. Wolf and Keigo Araki for discussion. This work was supported by The Naito Foundation.

\section{References}

[1] G. I. Evan and K. H. Vousden, "Proliferation, cell cycle and apoptosis in cancer," Nature, vol. 411, no. 6835, pp. 342-348, 2001.

[2] D. Hanahan and R. A. Weinberg, "Hallmarks of cancer: the next generation," Cell, vol. 144, no. 5, pp. 646-674, 2011.

[3] S. Heerboth, G. Housman, M. Leary et al., "EMT and tumor metastasis," Clinical and Translational Medicine, vol. 4, no. 1, article 6, 2015.

[4] S. H. Lee and R. Dominguez, "Regulation of actin cytoskeleton dynamics in cells," Molecules and Cells, vol. 29, no. 4, pp. 311-325, 2010.

[5] A. Nürnberg, T. Kitzing, and R. Grosse, "Nucleating actin for invasion," Nature Reviews Cancer, vol. 11, no. 3, pp. 117-187, 2011.

[6] M. D. Welch, J. Rosenblatt, J. Skoble, D. A. Portnoy, and T. J. Mitchison, "Interaction of human Arp2/3 complex and the Listeria monocytogenes ActA protein in actin filament nucleation," Science, vol. 281, no. 5373, pp. 105-108, 1998.

[7] M. E. Quinlan, J. E. Heuser, E. Kerkhoff, and R. D. Mullins, "Drosophila Spire is an actin nucleation factor," Nature, vol. 433, no. 7024, pp. 382-388, 2005.

[8] J. Faix and R. Grosse, "Staying in shape with formins," Developmental Cell, vol. 10, no. 6, pp. 693-706, 2006.

[9] C. D. Nobes and A. Hall, "Rho, Rac, and Cdc42 GTPases regulate the assembly of multimolecular focal complexes associated with actin stress fibers, lamellipodia, and filopodia," Cell, vol. 81, no. 1, pp. 53-62, 1995.

[10] F. S. Southwick, "Gelsolin and ADF/cofilin enhance the actin dynamics of motile cells," Proceedings of the National Academy of Sciences of the United States of America, vol. 97, no. 13, pp. 6936-6938, 2000.

[11] B. W. Bernstein and J. R. Bamburg, "ADF/Cofilin: a functional node in cell biology," Trends in Cell Biology, vol. 20, no. 4, pp. 187-195, 2010.

[12] J. Toshima, J. Y. Toshima, T. Amano, N. Yang, S. Narumiya, and K. Mizuno, "Cofilin phosphorylation by protein kinase testicular protein kinase 1 and its role in integrin-mediated actin reorganization and focal adhesion formation," Molecular Biology of the Cell, vol. 12, no. 4, pp. 1131-1145, 2001.

[13] Y.-B. Kim, S. Choi, M.-C. Choi et al., "Cell adhesion-dependent cofilin serine 3 phosphorylation by the integrin-linked kinase.cSrc complex," Journal of Biological Chemistry, vol. 283, no. 15, pp. 10089-10096, 2008.

[14] S. Linder, "The matrix corroded: podosomes and invadopodia in extracellular matrix degradation," Trends in Cell Biology, vol. 17, no. 3, pp. 107-117, 2007.

[15] L. M. Machesky, "Lamellipodia and filopodia in metastasis and invasion," FEBS Letters, vol. 582, no. 14, pp. 2102-2111, 2008.

[16] O. T. Fackler and R. Grosse, "Cell motility through plasma membrane blebbing," The Journal of Cell Biology, vol. 181, no. 6, pp. 879-884, 2008.

[17] G. Charras and E. Paluch, "Blebs lead the way: how to migrate without lamellipodia," Nature Reviews Molecular Cell Biology, vol. 9, no. 9, pp. 730-736, 2008.

[18] M. De Nicola, C. Cerella, M. D’Alessio et al., “The cleavage mode of apoptotic nuclear vesiculation is related to plasma membrane blebbing and depends on actin reorganization," Annals of the New York Academy of Sciences, vol. 1090, pp. 69-78, 2006.

[19] B. Geiger, J. P. Spatz, and A. D. Bershadsky, "Environmental sensing through focal adhesions," Nature Reviews Molecular Cell Biology, vol. 10, no. 1, pp. 21-33, 2009.

[20] J. Y.-J. Shyy and S. Chien, "Role of integrins in endothelial mechanosensing of shear stress," Circulation Research, vol. 91, no. 9, pp. 769-775, 2002.

[21] S. K. Mitra, D. A. Hanson, and D. D. Schlaepfer, "Focal adhesion kinase: in command and control of cell motility," Nature Reviews Molecular Cell Biology, vol. 6, no. 1, pp. 56-68, 2005.

[22] N. O. Deakin and C. E. Turner, "Paxillin comes of age," Journal of Cell Science, vol. 121, no. 15, pp. 2435-2444, 2008.

[23] S. Huveneers and E. H. J. Danen, "Adhesion signaling-crosstalk between integrins, Src and Rho," Journal of Cell Science, vol. 122, no. 8, pp. 1059-1069, 2009.

[24] S. V. Plotnikov, A. M. Pasapera, B. Sabass, and C. M. Waterman, "Force fluctuations within focal adhesions mediate ECMrigidity sensing to guide directed cell migration," Cell, vol. 151, no. 7, pp. 1513-1527, 2012.

[25] S. W. Moore, P. Roca-Cusachs, and M. P. Sheetz, "Stretchy proteins on stretchy substrates: the important elements of integrinmediated rigidity sensing," Developmental Cell, vol. 19, no. 2, pp. 194-206, 2010.

[26] S. Yonemura, Y. Wada, T. Watanabe, A. Nagafuchi, and M. Shibata, " $\alpha$-Catenin as a tension transducer that induces adherens junction development," Nature Cell Biology, vol. 12, no. 6, pp. 533-542, 2010

[27] M. Yao, W. Qiu, R. Liu et al., "Force-dependent conformational switch of $\alpha$-catenin controls vinculin binding," Nature Communications, vol. 5, article no. 4525, 2014.

[28] S. Huveneers and J. de Rooij, "Mechanosensitive systems at the cadherin-f-actin interface," Journal of Cell Science, vol. 126, no. 2, pp. 403-413, 2013.

[29] L. Wei, W. Zhou, J. D. Croissant et al., "RhoA signaling via serum response factor plays an obligatory role in myogenic differentiation," Journal of Biological Chemistry, vol. 273, no. 46, pp. 3028730294, 1998.

[30] A. L'Honore, N. J. Lamb, M. Vandromme, P. Turowski, G. Carnac, and A. Fernandez, "MyoD distal regulatory region contains an SRF binding CArG element required for MyoD 
expression in skeletal myoblasts and during muscle regeneration," Molecular Biology of the Cell, vol. 14, no. 5, pp. 2151-2162, 2003.

[31] G. C. T. Pipes, E. E. Creemers, and E. N. Olson, “The myocardin family of transcriptional coactivators: versatile regulators of cell growth, migration, and myogenesis," Genes and Development, vol. 20, no. 12, pp. 1545-1556, 2006.

[32] J.-R. Kim, H. J. Kee, J.-Y. Kim et al., "Enhancer of polycomb1 acts on serum response factor to regulate skeletal muscle differentiation," Journal of Biological Chemistry, vol. 284, no. 24, pp. 16308-16316, 2009.

[33] K. Kawauchi, W. W. Tan, K. Araki et al., "p130Cas-dependent actin remodelling regulates myogenic differentiation," Biochemical Journal, vol. 445, no. 3, pp. 323-332, 2012.

[34] N. Rivlin, R. Brosh, M. Oren, and V. Rotter, "Mutations in the p53 tumor suppressor gene: important milestones at the various steps of tumorigenesis," Genes and Cancer, vol. 2, no. 4, pp. 466474, 2011.

[35] K. H. Vousden and X. Lu, "Live or let die: the cell's response to p53," Nature Reviews Cancer, vol. 2, no. 8, pp. 594-604, 2002.

[36] M. Oren, "Decision making by p53: life, death and cancer," Cell Death and Differentiation, vol. 10, no. 4, pp. 431-442, 2003.

[37] K. H. Vousden and D. P. Lane, "p53 in health and disease," Nature Reviews Molecular Cell Biology, vol. 8, no. 4, pp. 275-283, 2007.

[38] E. Appella and C. W. Anderson, "Post-translational modifications and activation of p53 by genotoxic stresses," European Journal of Biochemistry, vol. 268, no. 10, pp. 2764-2772, 2001.

[39] D. W. Meek, "Tumour suppression by p53: a role for the DNA damage response?" Nature Reviews Cancer, vol. 9, no. 10, pp. 714-723, 2009.

[40] C. Giacinti and A. Giordano, "RB and cell cycle progression," Oncogene, vol. 25, no. 38, pp. 5220-5227, 2006.

[41] R. A. Weinberg, "The retinoblastoma protein and cell cycle control," Cell, vol. 81, no. 3, pp. 323-330, 1995.

[42] J. W. Harbour and D. C. Dean, "The Rb/E2F pathway: expanding roles and emerging paradigms," Genes and Development, vol. 14, no. 19, pp. 2393-2409, 2000.

[43] P. Viatour and J. Sage, "Newly identified aspects of tumor suppression by RB," Disease Models and Mechanisms, vol. 4, no. 5, pp. 581-585, 2011.

[44] K. Fukasawa, "Oncogenes and tumour suppressors take on centrosomes," Nature Reviews Cancer, vol. 7, no. 12, pp. 911-924, 2007.

[45] C. Brakebusch and R. Fässler, "The integrin-actin connection, an eternal love affair," The EMBO Journal, vol. 22, no. 10, pp. 2324-2333, 2003.

[46] J. S. Desgrosellier and D. A. Cheresh, "Integrins in cancer: biological implications and therapeutic opportunities," Nature Reviews Cancer, vol. 10, no. 1, pp. 9-22, 2010.

[47] S. Cabodi, M. Del Pilar Camacho-Leal, P. Di Stefano, and P. Defilippi, "Integrin signalling adaptors: not only figurants in the cancer story," Nature Reviews Cancer, vol. 10, no. 12, pp. 858870, 2010.

[48] S. Johansson, G. Svineng, K. Wennerberg, A. Armulik, and L. Lohikangas, "Fibronectin-integrin interactions," Frontiers in Bioscience, vol. 2, pp. d126-d146, 1997.

[49] M. M. Zutter, S. A. Santoro, W. D. Staatz, and Y. L. Tsung, "Reexpression of the $\alpha 2 \beta 1$ integrin abrogates the malignant phenotype of breast carcinoma cells," Proceedings of the National
Academy of Sciences of the United States of America, vol. 92, no. 16, pp. 7411-7415, 1995.

[50] A. Kren, V. Baeriswyl, F. Lehembre et al., "Increased tumor cell dissemination and cellular senescence in the absence of $\beta_{1}$ integrin function," The EMBO Journal, vol. 26, no. 12, pp. 2832$2842,2007$.

[51] H. Janouskova, A. Maglott, D. Y. Leger et al., "Integrin $\alpha 5 \beta 1$ plays a critical role in resistance to temozolomide by interfering with the p53 pathway in high-grade glioma," Cancer Research, vol. 72, no. 14, pp. 3463-3470, 2012.

[52] H. Janouskova, A.-M. Ray, F. Noulet et al., "Activation of p53 pathway by Nutlin-3a inhibits the expression of the therapeutic target $\alpha 5$ integrin in colon cancer cells," Cancer Letters, vol. 336, no. 2, pp. 307-318, 2013.

[53] J. Qiu, G. Wang, J. Hu, Q. Peng, and Y. Zheng, "Id1-induced inhibition of p53 facilitates endothelial cell migration and tube formation by regulating the expression of betal-integrin," Molecular and Cellular Biochemistry, vol. 357, no. 1-2, pp. 125-133, 2011.

[54] F. Vaillant, M.-L. Asselin-Labat, M. Shackleton, N. C. Forrest, G. J. Lindeman, and J. E. Visvader, "The mammary progenitor marker CD61/ $\beta 3$ integrin identifies cancer stem cells in mouse models of mammary tumorigenesis," Cancer Research, vol. 68, no. 19, pp. 7711-7717, 2008.

[55] A. K. Guo, Y. Y. Hou, H. Hirata et al., "Loss of p53 enhances NF- $\kappa$ B-dependent lamellipodia formation," Journal of Cellular Physiology, vol. 229, no. 6, pp. 696-704, 2014.

[56] G. Bon, S. E. Di Carlo, V. Folgiero et al., "Negative regulation of $\beta 4$ integrin transcription by homeodomain-interacting protein kinase 2 and p53 impairs tumor progression," Cancer Research, vol. 69, no. 14, pp. 5978-5986, 2009.

[57] Y. Sawada, M. Tamada, B. J. Dubin-Thaler et al., "Force Sensing by Mechanical Extension of the Src Family Kinase Substrate p130Cas," Cell, vol. 127, no. 5, pp. 1015-1026, 2006.

[58] Z. Zhao, S. H. Tan, H. Machiyama et al., "Association between tensin 1 and p130Cas at focal adhesions links actin inward flux to cell migration," Biology Open, vol. 5, no. 4, pp. 499-506, 2016.

[59] H. Chen, A. Ishii, W.-K. Wong, L. B. Chen, and S. H. Lo, "Molecular characterization of human tensin," Biochemical Journal, vol. 351, no. 2, pp. 403-411, 2000.

[60] D. R. Rhodes, J. Yu, K. Shanker et al., "ONCOMINE: a cancer microarray database and integrated data-mining platform," Neoplasia, vol. 6, no. 1, pp. 1-6, 2004.

[61] T. Watanabe-Nakayama, M. Saito, S. Machida, K. Kishimoto, R. Afrin, and A. Ikai, "Requirement of LIM domains for the transient accumulation of paxillin at damaged stress fibres," Biology Open, vol. 2, no. 7, pp. 667-674, 2013.

[62] M. C. Brown and C. E. Turner, "Paxillin: adapting to change," Physiological Reviews, vol. 84, no. 4, pp. 1315-1339, 2004.

[63] V. Golubovskaya, A. Kaur, and W. Cance, "Cloning and characterization of the promoter region of human focal adhesion kinase gene: nuclear factor kappa B and p53 binding sites," Biochimica et Biophysica Acta (BBA)-Gene Structure and Expression, vol. 1678, no. 2-3, pp. 111-125, 2004.

[64] C. Tanikawa, Y. Furukawa, N. Yoshida, H. Arakawa, Y. Nakamura, and K. Matsuda, "XEDAR as a putative colorectal tumor suppressor that mediates p53-regulated anoikis pathway," Oncogene, vol. 28, no. 34, pp. 3081-3092, 2009.

[65] S. Yamauchi, Y. Y. Hou, A. K. Guo et al., "p53-mediated activation of the mitochondrial protease HtrA2/Omi prevents cell invasion," Journal of Cell Biology, vol. 204, no. 7, pp. 1191-1207, 2014. 
[66] A. Sharma and B. J. Mayer, "Phosphorylation of p130Cas initiates Rac activation and membrane ruffling," BMC Cell Biology, vol. 9, article 50, 2008.

[67] H. Plun-Favreau, K. Klupsch, N. Moisoi et al., "The mitochondrial protease HtrA2 is regulated by Parkinson's diseaseassociated kinase PINK1," Nature Cell Biology, vol. 9, no. 11, pp. 1243-1252, 2007.

[68] D. R. Green and G. Kroemer, "Cytoplasmic functions of the tumour suppressor p53," Nature, vol. 458, no. 7242, pp. 11271130, 2009.

[69] M. Aga, J. M. Bradley, K. E. Keller, M. J. Kelley, and T. S. Acott, "Specialized podosome-or invadopodia-like structures (PILS) for focal trabecular meshwork extracellular matrix turnover," Investigative Ophthalmology and Visual Science, vol. 49, no. 12, pp. 5353-5365, 2008.

[70] U. K. Mukhopadhyay, R. Eves, L. Jia, P. Mooney, and A. S. Mak, "p53 suppresses Src-induced podosome and rosette formation and cellular invasiveness through the upregulation of caldesmon," Molecular and Cellular Biology, vol. 29, no. 11, pp. 3088-3098, 2009.

[71] U. K. Mukhopadhyay, P. Mooney, L. Jia, R. Eves, L. Raptis, and A. S. Mak, "Doubles game: Src-Stat3 versus p53-PTEN in cellular migration and invasion," Molecular and Cellular Biology, vol. 30, no. 21, pp. 4980-4995, 2010.

[72] T. Yoshio, T. Morita, Y. Kimura, M. Tsujii, N. Hayashi, and K. Sobue, "Caldesmon suppresses cancer cell invasion by regulating podosome/invadopodium formation," FEBS Letters, vol. 581, no. 20, pp. 3777-3782, 2007.

[73] P. A. J. Muller, K. H. Vousden, and J. C. Norman, "p53 and its mutants in tumor cell migration and invasion," Journal of Cell Biology, vol. 192, no. 2, pp. 209-218, 2011.

[74] E. Powell, D. Piwnica-Worms, and H. Piwnica-Worms, "Contribution of p53 to metastasis," Cancer Discovery, vol. 4, no. 4, pp. 405-414, 2014.

[75] K. Araki, T. Ebata, A. K. Guo, K. Tobiume, S. J. Wolf, and K. Kawauchi, "P53 regulates cytoskeleton remodeling to suppress tumor progression," Cellular and Molecular Life Sciences, vol. 72, no. 21, pp. 4077-4094, 2015.

[76] M. Xia and H. Land, "Tumor suppressor p53 restricts Ras stimulation of RhoA and cancer cell motility," Nature Structural and Molecular Biology, vol. 14, no. 3, pp. 215-223, 2007.

[77] G. Gadea, M. de Toledo, C. Anguille, and P. Roux, "Loss of p53 promotes RhoA-ROCK-dependent cell migration and invasion in 3D matrices," The Journal of Cell Biology, vol. 178, no. 1, pp. 23-30, 2007.

[78] K. Lefort, A. Mandinova, P. Ostano et al., "Notch1 is a p53 target gene involved in human keratinocyte tumor suppression through negative regulation of ROCK1/2 and MRCK $\alpha$ kinases," Genes and Development, vol. 21, no. 5, pp. 562-577, 2007.

[79] F. Guo, Y. Gao, L. Wang, and Y. Zheng, "p19 ${ }^{\text {Arf }}$-p53 tumor suppressor pathway regulates cell motility by suppression of phosphoinositide 3-kinase and Racl GTPase activities," Journal of Biological Chemistry, vol. 278, no. 16, pp. 14414-14419, 2003.

[80] G. Gadéa, L. Lapasset, C. Gauthier-Rouvière, and P. Roux, "Regulation of Cdc42-mediated morphological effects: a novel function for p53," EMBO Journal, vol. 21, no. 10, pp. 2373-2382, 2002.

[81] D. R. Croft, D. Crighton, M. S. Samuel et al., "p53-mediated transcriptional regulation and activation of the actin cytoskeleton regulatory RhoC to LIMK2 signaling pathway promotes cell survival," Cell Research, vol. 21, no. 4, pp. 666-682, 2011.
[82] P. P. Ongusaha, H.-G. Kim, S. A. Boswell et al., "RhoE is a pro-survival p53 target gene that inhibits ROCK I-mediated apoptosis in response to genotoxic stress," Current Biology, vol. 16, no. 24, pp. 2466-2472, 2006.

[83] A. E. Hall, W. Lu, J. D. Godfrey et al., “The cytoskeleton adaptor protein ankyrin-1 is upregulated by $\mathrm{p} 53$ following DNA damage and alters cell migration," Cell Death and Disease, vol. 7, no. 4, Article ID e2184, 2016.

[84] V. Bennett and A. J. Baines, "Spectrin and ankyrin-based pathways: metazoan inventions for integrating cells into tissues," Physiological Reviews, vol. 81, no. 3, pp. 1353-1392, 2001.

[85] V. Bennett and J. Healy, "Membrane domains based on ankyrin and spectrin associated with cell-cell interactions," Cold Spring Harbor Perspectives in Biology, vol. 1, no. 6, Article ID a003012, 2009.

[86] P. R. Stabach, I. Simonović, M. A. Ranieri et al., "The structure of the ankyrin-binding site of $\beta$-spectrin reveals how tandem spectrin-repeats generate unique ligand-binding properties," Blood, vol. 113, no. 22, pp. 5377-5384, 2009.

[87] A. Nagafuchi, "Molecular architecture of adherens junctions," Current Opinion in Cell Biology, vol. 13, no. 5, pp. 600-603, 2001.

[88] A. R. Harris, A. Daeden, and G. T. Charras, "Formation of adherens junctions leads to the emergence of a tissue-level tension in epithelial monolayers," Journal of Cell Science, vol. 127, no. 11, pp. 2507-2517, 2014.

[89] T. Lecuit and A. S. Yap, "E-cadherin junctions as active mechanical integrators in tissue dynamics," Nature Cell Biology, vol. 17, no. 5, pp. 533-539, 2015.

[90] B. Ladoux, W. J. Nelson, J. Yan, and R. M. Mège, "The mechanotransduction machinery at work at adherens junctions," Integrative Biology, vol. 7, no. 10, pp. 1109-1119, 2015.

[91] G. Berx and F. van Roy, "Involvement of members of the cadherin superfamily in cancer," Cold Spring Harbor Perspectives in Biology, vol. 1, no. 6, Article ID a003129, 2009.

[92] H. Siemens, R. Jackstadt, S. Hünten et al., "miR-34 and SNAIL form a double-negative feedback loop to regulate epithelialmesenchymal transitions," Cell Cycle, vol. 10, no. 24, pp. 42564271, 2011.

[93] C.-J. Chang, C.-H. Chao, W. Xia et al., "P53 regulates epithelialmesenchymal transition and stem cell properties through modulating miRNAs," Nature Cell Biology, vol. 13, no. 3, pp. 317-323, 2011.

[94] T. Kim, A. Veronese, F. Pichiorri et al., "p53 regulates epithelialmesenchymal transition through microRNAs targeting ZEB1 and ZEB2," Journal of Experimental Medicine, vol. 208, no. 5, pp. 875-883, 2011.

[95] S.-P. Wang, W.-L. Wang, Y.-L. Chang et al., "p53 controls cancer cell invasion by inducing the MDM2-mediated degradation of Slug," Nature Cell Biology, vol. 11, no. 6, pp. 694-704, 2009.

[96] J. Kim, S. Bae, S. An et al., "Cooperative actions of p21WAF1 and p53 induce Slug protein degradation and suppress cell invasion," EMBO Reports, vol. 15, no. 10, pp. 1062-1068, 2014.

[97] P. A. J. Muller and K. H. Vousden, "P53 mutations in cancer," Nature Cell Biology, vol. 15, no. 1, pp. 2-8, 2013.

[98] P. A. J. Muller, P. T. Caswell, B. Doyle et al., "Mutant p53 drives invasion by promoting integrin recycling," Cell, vol. 139, no. 7, pp. 1327-1341, 2009.

[99] A. Arjonen, R. Kaukonen, E. Mattila et al., "Mutant p53.associated myosin-X upregulation promotes breast cancer invasion and metastasis," Journal of Clinical Investigation, vol. 124, no. 3, pp. 1069-1082, 2014. 
[100] M. P. Iwanicki, H. Y. Chen, C. Iavarone et al., "Mutant p53 regulates ovarian cancer transformed phenotypes through autocrine matrix deposition," JCI Insight, vol. 1, no. 10, 2016.

[101] J.-G. Lee, J.-H. Ahn, T. J. Kim, J. H. Lee, and J.-H. Choi, “Mutant p53 promotes ovarian cancer cell adhesion to mesothelial cells via integrin $\beta 4$ and Akt signals," Scientific Reports, vol. 5, Article ID 12642, 2015.

[102] Y. Li and C. Prives, "Are interactions with p63 and p73 involved in mutant p53 gain of oncogenic function?" Oncogene, vol. 26, no. 15, pp. 2220-2225, 2007.

[103] Y. Zhang, W. Yan, and X. Chen, "Mutant p53 disrupts MCF$10 \mathrm{~A}$ cell polarity in three-dimensional culture via epithelial-tomesenchymal transitions," Journal of Biological Chemistry, vol. 286, no. 18, pp. 16218-16228, 2011.

[104] I. Kogan-Sakin, Y. Tabach, Y. Buganim et al., "Mutant p53(R175H) upregulates Twistl expression and promotes epithelial-mesenchymal transition in immortalized prostate cells," Cell Death \& Differentiation, vol. 18, no. 2, pp. 271-281, 2011.

[105] G. Bossi, F. Marampon, R. Maor-Aloni et al., "Conditional RNA interference in vivo to study mutant p53 oncogenic gain of function on tumor malignancy," Cell Cycle, vol. 7, no. 12, pp. 1870-1879, 2008.

[106] B. E. Engel, E. Welsh, M. F. Emmons, P. G. Santiago-Cardona, and W. D. Cress, "Expression of integrin alpha 10 is transcriptionally activated by $\mathrm{pRb}$ in mouse osteoblasts and is downregulated in multiple solid tumors," Cell Death and Disease, vol. 4, no. 11, article e938, 2013.

[107] M. Barczyk, S. Carracedo, and D. Gullberg, "Integrins," Cell and Tissue Research, vol. 339, no. 1, pp. 269-280, 2010.

[108] W. K. A. Sikkema, A. Strikwerda, M. Sharma et al., "Regulation of mitotic cytoskeleton dynamics and cytokinesis by integrinlinked kinase in retinoblastoma cells," PLoS ONE, vol. 9, no. 6, Article ID e98838, 2014.

[109] N. R. Filipenko, S. Attwell, C. Roskelley, and S. Dedhar, "Integrin-linked kinase activity regulates Rac- and Cdc42mediated actin cytoskeleton reorganization via $\alpha$-PIX," Oncogene, vol. 24, no. 38, pp. 5837-5849, 2005.

[110] A. B. Fielding, I. Dobreva, and S. Dedhar, "Beyond focal adhesions: integrin-linked kinase associates with tubulin and regulates mitotic spindle organization," Cell Cycle, vol. 7, no. 13, pp. 1899-1906, 2008.

[111] A. B. Fielding, S. Lim, K. Montgomery, I. Dobreva, and S. Dedhar, "A critical role of integrin-linked kinase, ch-TOG and TACC3 in centrosome clustering in cancer cells," Oncogene, vol. 30, no. 5, pp. 521-534, 2011.

[112] M. Kwon, S. A. Godinho, N. S. Chandhok et al., "Mechanisms to suppress multipolar divisions in cancer cells with extra centrosomes," Genes and Development, vol. 22, no. 16, pp. 21892203, 2008.

[113] K. Araki, K. Kawauchi, H. Hirata, M. Yamamoto, and Y. Taya, "Cytoplasmic translocation of the retinoblastoma protein disrupts sarcomeric organization," eLife, vol. 2, Article ID e01228, 2013.

[114] A. Mammoto, S. Huang, K. Moore, P. Oh, and D. E. Ingber, "Role of RhoA, mDia, and ROCK in cell shape-dependent control of the Skp2-p27kip-1 pathway and the G1/S transition," Journal of Biological Chemistry, vol. 279, no. 25, pp. 2632326330, 2004.

[115] B. Sosa-García, V. Gunduz, V. Vazquez-Rivera et al., "A role for the retinoblastoma protein as a regulator of mouse osteoblast cell adhesion: implications for osteogenesis and osteosarcoma formation," PLOS ONE, vol. 5, no. 11, Article ID e13954, 2010.
[116] R. G. Fehon, A. I. McClatchey, and A. Bretscher, "Organizing the cell cortex: the role of ERM proteins," Nature Reviews Molecular Cell Biology, vol. 11, no. 4, pp. 276-287, 2010.

[117] K. Tomita, A. Van Bokhoven, G. J. L. H. Van Leenders et al., "Cadherin switching in human prostate cancer progression," Cancer Research, vol. 60, no. 13, pp. 3650-3654, 2000.

[118] G. Nakajima, A. Patino-Garcia, S. Bruheim et al., "CDH11 expression is associated with survival in patients with osteosarcoma," Cancer Genomics and Proteomics, vol. 5, no. 1, pp. 37-42, 2008.

[119] D. Tamura, T. Hiraga, A. Myoui, H. Yoshikawa, and T. Yoneda, "Cadherin-11-mediated interactions with bone marrow stromal/osteoblastic cells support selective colonization of breast cancer cells in bone," International Journal of Oncology, vol. 33, no. 1, pp. 17-24, 2008.

[120] K. Chu, C.-J. Cheng, X. Ye et al., "Cadherin-11 promotes the metastasis of prostate cancer cells to bone," Molecular Cancer Research, vol. 6, no. 8, pp. 1259-1267, 2008.

[121] S. Floor, W. C. G. Van Staveren, D. Larsimont, J. E. Dumont, and C. Maenhaut, "Cancer cells in epithelial-to-mesenchymal transition and tumor-propagating-cancer stem cells: distinct, overlapping or same populations," Oncogene, vol. 30, no. 46, pp. 4609-4621, 2011.

[122] H. Kaur, P. J. Phillips-Mason, S. M. Burden-Gulley et al., "Cadherin-11, a marker of the mesenchymal phenotype, regulates glioblastoma cell migration and survival in vivo," Molecular Cancer Research, vol. 10, no. 3, pp. 293-304, 2012.

[123] S. Kumar and V. M. Weaver, "Mechanics, malignancy, and metastasis: the force journey of a tumor cell," Cancer and Metastasis Reviews, vol. 28, no. 1-2, pp. 113-127, 2009.

[124] D. Wirtz, K. Konstantopoulos, and P. C. Searson, "The physics of cancer: the role of physical interactions and mechanical forces in metastasis," Nature Reviews Cancer, vol. 11, no. 7, pp. 512-522, 2011.

[125] M. J. Paszek, N. Zahir, K. R. Johnson et al., "Tensional homeostasis and the malignant phenotype," Cancer Cell, vol. 8, no. 3, pp. 241-254, 2005.

[126] K. R. Levental, H. Yu, L. Kass et al., "Matrix crosslinking forces tumor progression by enhancing integrin signaling," Cell, vol. 139, no. 5, pp. 891-906, 2009.

[127] J. Wels, R. N. Kaplan, S. Rafii, and D. Lyden, "Migratory neighbors and distant invaders: tumor-associated niche cells," Genes and Development, vol. 22, no. 5, pp. 559-574, 2008.

[128] H. Ungefroren, S. Sebens, D. Seidl, H. Lehnert, and R. Hass, "Interaction of tumor cells with the microenvironment," Cell Communication and Signaling, vol. 9, article no. 18, 2011.

[129] P. Lu, V. M. Weaver, and Z. Werb, “The extracellular matrix: a dynamic niche in cancer progression," Journal of Cell Biology, vol. 196, no. 4, pp. 395-406, 2012.

[130] S. Valastyan and R. A. Weinberg, "Tumor metastasis: molecular insights and evolving paradigms," Cell, vol. 147, no. 2, pp. 275292, 2011.

[131] M. Castells, B. Thibault, J.-P. Delord, and B. Couderc, "Implication of tumor microenvironment in chemoresistance: tumorassociated stromal cells protect tumor cells from cell death," International Journal of Molecular Sciences, vol. 13, no. 8, pp. 9545-9571, 2012.

[132] M. Schwarzkopf, D. Coletti, D. Sassoon, and G. Marazzi, "Muscle cachexia is regulated by a p53-PW1/Peg3-dependent pathway," Genes and Development, vol. 20, no. 24, pp. 3440-3452, 2006. 

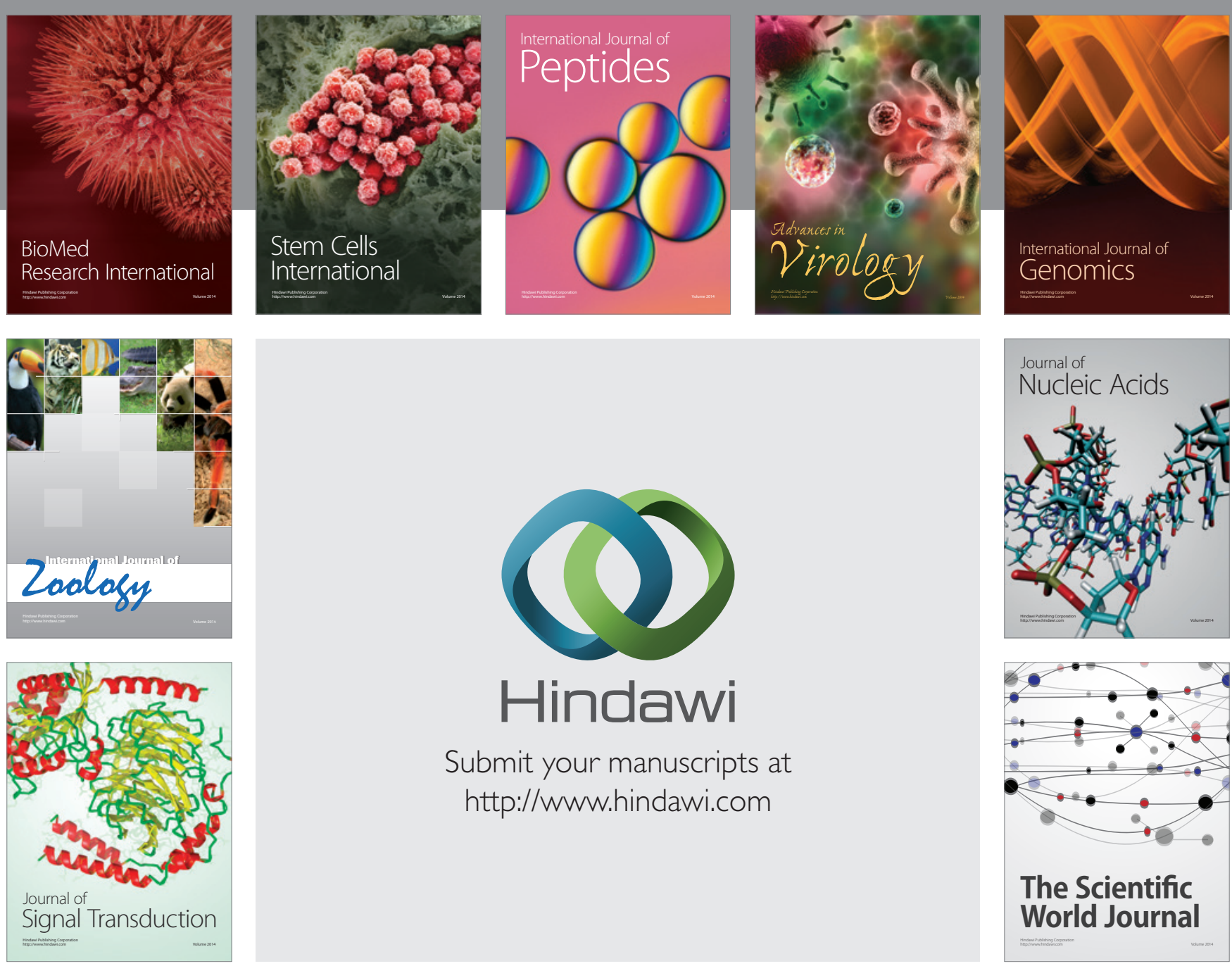

Submit your manuscripts at

http://www.hindawi.com
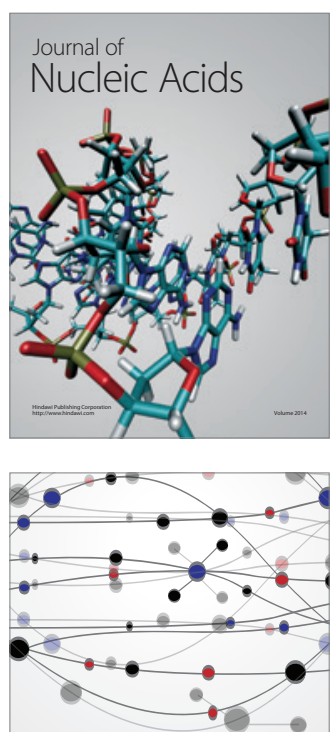

The Scientific World Journal
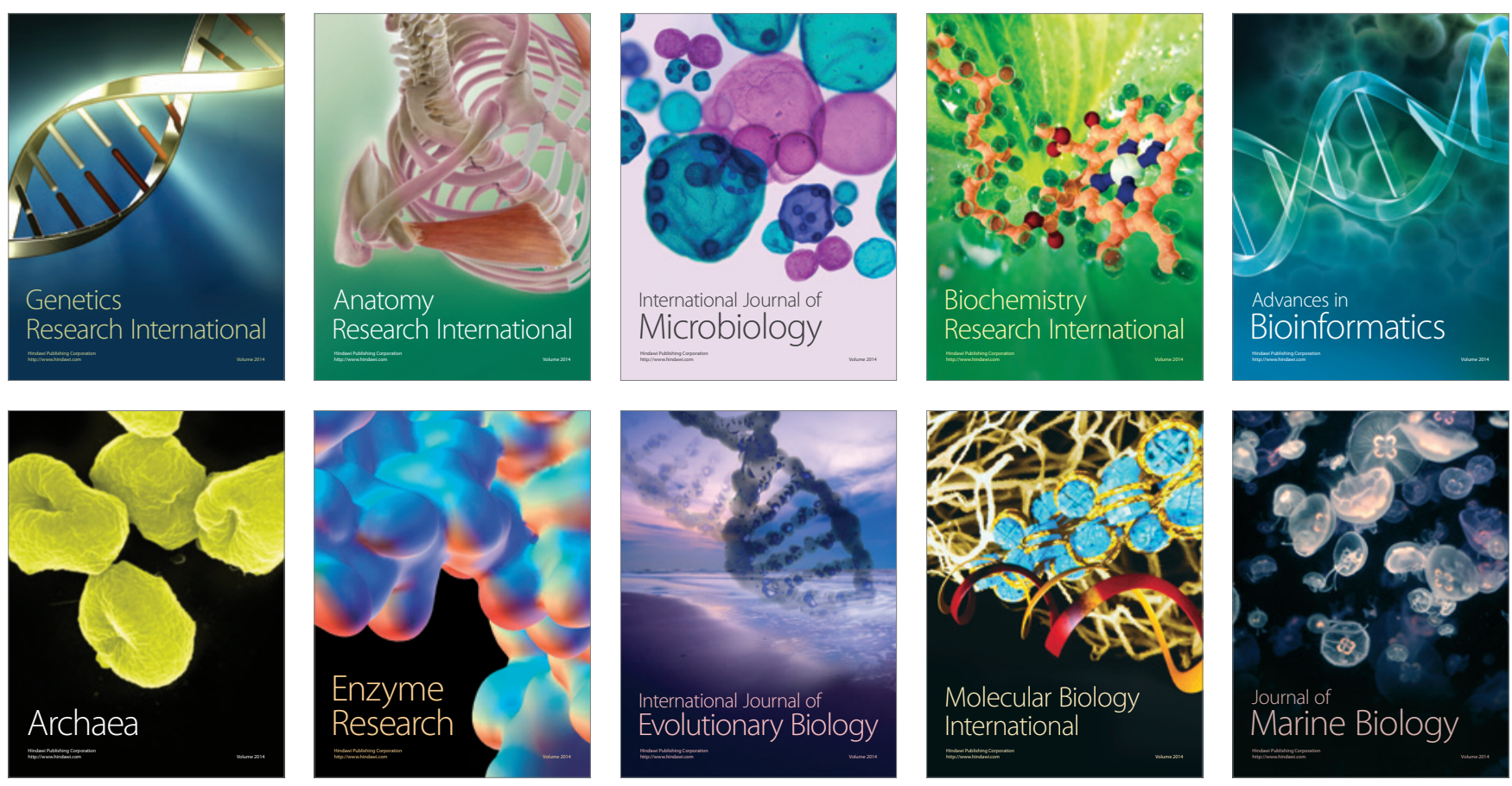